Provided for non-commercial research and educational use only. Not for reproduction or distribution or commercial use.

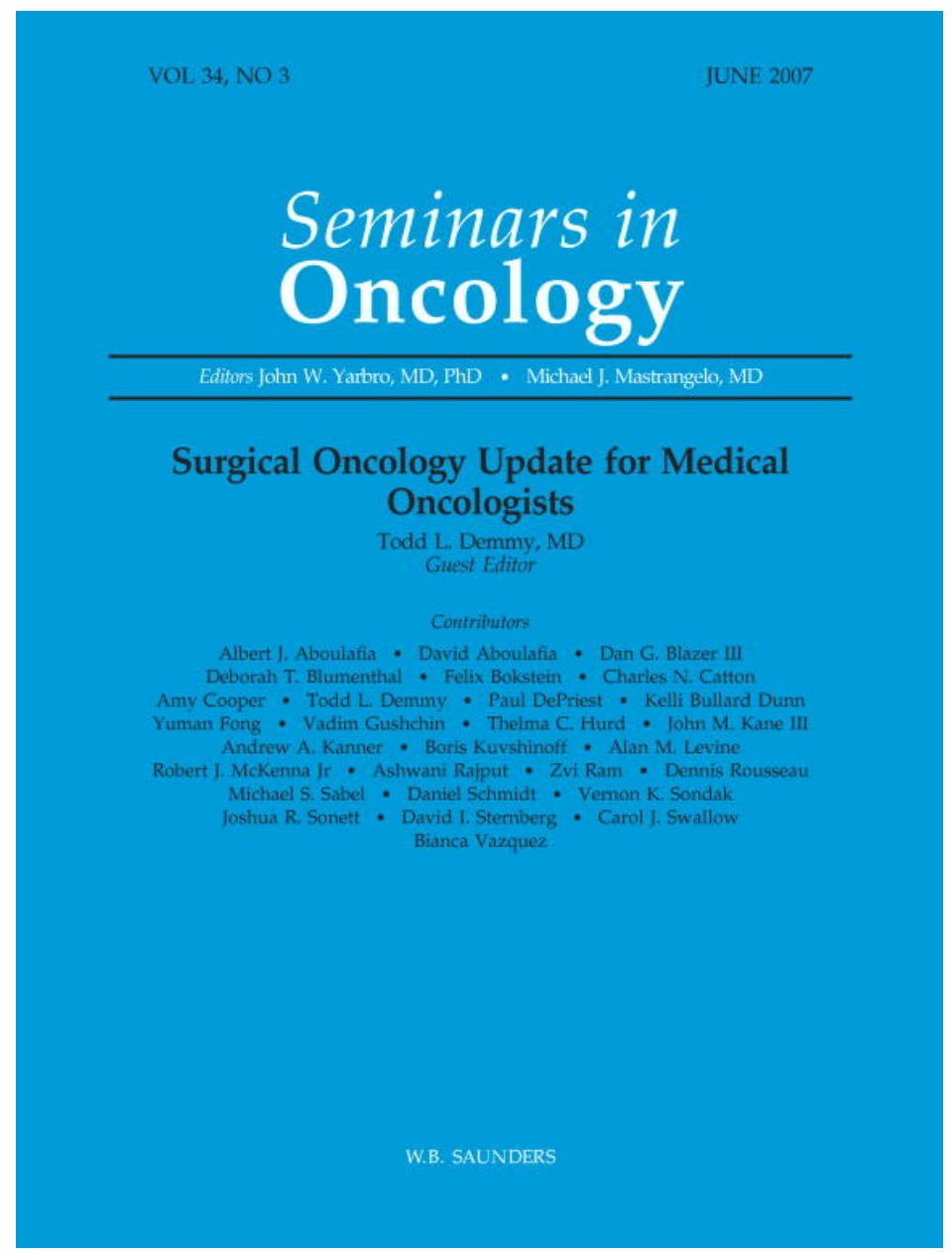

This article was originally published in a journal published by Elsevier, and the attached copy is provided by Elsevier for the author's benefit and for the benefit of the author's institution, for non-commercial research and educational use including without limitation use in instruction at your institution, sending it to specific colleagues that you know, and providing a copy to your institution's administrator.

All other uses, reproduction and distribution, including without limitation commercial reprints, selling or licensing copies or access,

or posting on open internet sites, your personal or institution's website or repository, are prohibited. For exceptions, permission may be sought for such use through Elsevier's permissions site at: 


\title{
Surgical Therapy of Cutaneous Melanoma
}

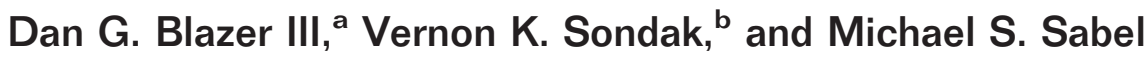

\begin{abstract}
For most solid tumors therapy has evolved from surgery alone to a multidisciplinary approach. Malignant melanoma remains an exception, with surgery maintaining the principal role not only for treatment of the primary lesion but also staging and the management of advanced disease. The surgical management of melanoma has evolved over the years, resulting in a substantial decrease in the morbidity associated with treatment without a compromise in outcome. This article will review the changes that have occurred leading to the current surgical approach to melanoma, the evidence behind these recommendations, and new questions that need to be addressed.
\end{abstract}

Semin Oncol 34:270-280 @ 2007 Elsevier Inc. All rights reserved.

$\mathrm{W}$ ith an estimated 62,190 cases in the United States in 2006, cutaneous melanoma is expected to be the fifth most common cancer in men and the sixth most common cancer in women. ${ }^{1}$ The greatest impact that can be made on this disease is early diagnosis through careful surveillance and an aggressive use of biopsy for suspicious lesions. Survival is dramatically better when melanoma is discovered early before the lesion has thickened or spread to regional lymph nodes.

The treatment of many solid tumors has evolved over the years from surgery alone to multidisciplinary therapy. However, the management of melanoma remains centered on resection. Although chemotherapy and radiation therapy have made major contributions to the management of other malignancies, their benefit in melanoma has been limited. Biologic and immunologic therapies, while holding great promise, have yet to make a significant impact on melanoma outcome. Thus, surgical extirpation remains the best hope for long-term survival. This point is true not only for early-stage disease, but also advanced regional and, in some cases, distant disease. In this issue of Seminars in Oncology, we review the role of surgery in the treatment of melanoma.

${ }^{a}$ Division of Surgical Oncology, Department of Surgery, University of Michigan, Ann Arbor, MI

bDivision of Cutaneous Oncology, Department of Interdisciplinary Oncology, H. Lee Moffitt Cancer Center, University of South Florida, Tampa, FL.

Address correspondence to Michael S. Sabel, MD, University of Michigan Health System, 3304 Cancer Geriatric Center/0932, 1500 E Medical Center Dr, Ann Arbor, MI 48109. E-mail: msabel@umich.edu

\section{Excision of the Primary Melanoma}

Up to $85 \%$ of patients with newly diagnosed cutaneous melanoma have disease that is clinically confined to the site of the primary lesion. ${ }^{2}$ Because local recurrence rates associated with narrow but negative margins approach $60 \%,{ }^{3}$ excision with wide margins of normal skin has been the cornerstone of the surgical management of primary melanoma for 150 years, a strategy first described by William Norris in $1857 .{ }^{4}$

Although still a standard approach, the nature of the wide excision has evolved over time. In 1907 Handley advocated margins of 1 inch of skin and 2 inches of subcutaneous tissue in order to prevent recurrence. ${ }^{4}$ In the latter half of the 20th century, wider margins ( 4 or $5 \mathrm{~cm}$ ) were recommended based on the discovery of melanocytes or microsatellites beyond the recommended margins of excision. These radical excisions remained the standard of care in the treatment of melanoma for decades. However, the uncertainty of whether or not the substantial morbidity of these wider margins really did improve survival prompted studies to support these recommendations. Several randomized trials were initiated to test the necessity of wide $(3,4$, or $5 \mathrm{~cm})$ margins over narrow (1 or $2 \mathrm{~cm}$ ) margins (Table 1). Current recommendations are based on these trials, but the trials differed greatly in both the patient populations studied and the margins examined. Thus, while these trials taught us much about melanoma and thereby decreased treatment morbidity, the minimum adequate margin of excision is still uncertain.

The first randomized trial evaluating surgical margins for melanomas less than $2 \mathrm{~mm}$ thick was the World Health Organization (WHO) Melanoma Group study. ${ }^{5}$ This trial compared 1 -cm versus $3-\mathrm{cm}$ margins of excision in 612 patients. Long-term results demonstrated no survival differences be- 
Table 1 Randomized Trials of Narrow Versus Wide Excision Margins

\begin{tabular}{|c|c|c|c|c|c|}
\hline Trial & $\mathbf{N}$ & $\begin{array}{c}\text { Narrow } \\
\text { Margin } \\
\text { (cm) }\end{array}$ & $\begin{array}{l}\text { Wide } \\
\text { Margin } \\
\text { (cm) }\end{array}$ & $\begin{array}{c}\text { Tumor } \\
\text { Thickness } \\
\text { (mm) }\end{array}$ & Outcomes \\
\hline WHO Melanoma Program, $1991^{5}$ & 612 & 1 & 3 & $<2$ & NSD OS, DFS, LRR \\
\hline Intergroup Melanoma Surgical Trial, 19966,7 & 470 & 2 & 4 & $1-4$ & NSD OS, LRR \\
\hline Swedish Melanoma Group, $2000^{8}$ & 989 & 2 & 5 & $0.8-2$ & NSD OS, DFS, LRR \\
\hline French Cooperative Group, $2003^{9}$ & 326 & 2 & 5 & $<2.1$ & NSD OS, DFS \\
\hline United Kingdom Melanoma Group, $2004^{10}$ & 900 & 1 & 3 & $\geq 2$ & $\begin{array}{l}\text { NSD OS } \\
\text { Increased LRR with } 1-\mathrm{cm} \text { margins } \\
\quad(H R=1.26, P=.05) .\end{array}$ \\
\hline
\end{tabular}

Abbreviations: NSD, no significant difference; OS, overall survival; DFS, disease-free survival; LRR, local-regional recurrence; HR, hazard ratio.

tween these two patient groups. For the patients with melanomas $\leq 1 \mathrm{~mm}$, there were no local recurrences regardless of margin size. This trial thereby established the safety of $1-\mathrm{cm}$ margins of excision for thin melanomas. For patients with melanomas between 1 and $2 \mathrm{~mm}$, there was a 2.7\% local recurrence rate for 1 -cm excision margins compared with $0 \%$ in the 3-cm group. While this result is not statistically significant and had no survival impact, the study had insufficient statistical power to dispel concerns regarding 1 -cm excision margins for melanomas greater than $1 \mathrm{~mm}$ of depth.

The next three trials compared wider ( 4 or $5 \mathrm{~cm}$ ) margins of excision to 2-cm margins for intermediate thickness melanomas. The Intergroup Melanoma Surgical Trial ${ }^{6,7}$ enrolled 740 patients with intermediate-thickness ( 1 to $4 \mathrm{~mm}$ ) melanomas and randomly assigned 470 of these patients to either 2- or 4-cm resection margins. No statistically significant differences in survival or local recurrence were identified, even at 10 years. The Swedish Melanoma Study Group ${ }^{8}$ evaluated 989 patients with primary melanomas between 0.8 and 2.0 $\mathrm{mm}$ thickness. These patients were randomly assigned to excision with a 2 - or 5-cm margin, and again there were no statistically significant differences in local recurrence or survival rates. Finally, the French Cooperative Group 9 evaluated 326 patients with melanomas of $\leq 2 \mathrm{~mm}$ thickness and randomly assigned them to 2 - or 5-cm margins. This trial also demonstrated no differences in local recurrence or survival rates. Based on these study results, it is evident that margins greater than $2 \mathrm{~cm}$ are unnecessary for the management of intermediate thickness melanoma.

The British Cooperative Group Trial ${ }^{10}$ compared 1- versus 3-cm margins in 900 patients with melanoma thickness $\geq 2$ $\mathrm{mm}$. This randomized trial was the only one that included patients with melanomas $>4 \mathrm{~mm}$ thick, constituting $25 \%$ of the total group. No statistically significant difference in overall survival was observed. However, a $1-\mathrm{cm}$ excision margin was associated with a significantly increased rate of localregional recurrence, the majority of which occurred in the regional lymph nodes. There was also a trend, although not significant, towards decreased survival in the 1-cm margin group. Based on these results, the authors recommended margins of excision wider than $1 \mathrm{~cm}$ for melanomas greater than $2 \mathrm{~mm}$.

Cumulatively, these trials established the fact that margins of excision greater than $3 \mathrm{~cm}$ were unnecessary for the resec- tion of primary melanoma. They demonstrate that 1-cm margins of excision are adequate for thin $(\leq 1 \mathrm{~mm})$ melanomas, and $2-\mathrm{cm}$ margins are appropriate for melanomas $>1 \mathrm{~mm}$. However, are 2-cm margins necessary for melanomas greater than $1 \mathrm{~mm}$ in thickness, or would a 1-cm margin suffice? The argument for $2-\mathrm{cm}$ margins centers on two subgroups of the clinical trials:

- In the WHO trial melanomas between 1 and $2 \mathrm{~mm}$ had a $2.7 \%$ local recurrence rate when excised with only $1-\mathrm{cm}$ margins, compared to no local recurrences for 3-cm margins.

- In the British Cooperative Trial locoregional recurrence was significantly higher in the 1-cm margin group than the $3-\mathrm{cm}$ margin group, with a trend towards improved survival.

Does this current level of evidence support 2-cm margins? Although there were some local recurrences in the WHO trial for melanomas between 1 and $2 \mathrm{~mm}$ when only a 1-cm margin of excision was used, the difference was not statistically significant. In addition, there was no difference in overall survival between the two groups. For this reason, current guidelines recommend obtaining 2-cm margins (when possible) for melanomas between 1 and $2 \mathrm{~mm}$, but the margins may be decreased to as small as $1 \mathrm{~cm}$ if a skin graft or excessive closure tension can be avoided.

Stronger evidence for requiring a 2-cm margin for melanomas $>2 \mathrm{~mm}$ and, when possible, for melanomas between 1 and $2 \mathrm{~mm}$ comes from the British trial. Although there was a significant increase in local-regional recurrences with 1-cm margins, this result only achieved statistical significance when local and regional recurrences were added together. If one looks only at the local recurrence rate, there was no difference between the 2 treatment arms. Most of the recurrences were, in fact, regional. However, the trial was limited to wide local excision alone; patients undergoing elective lymph node dissection or sentinel lymph node (SLN) biopsy were not included. We now need to consider how these trial results might have changed if the current routine practice of SLN biopsy had been used during these studies.

Proponents of the 2-cm margin argue that the wider excision in the British trial, by incorporating the microscopic disease that was present outside of the $1-\mathrm{cm}$ radius, prevented this disease from ultimately reaching the lymph 
Table 2 Recommended Surgical Margins for Excision of Primary Melanoma

\begin{tabular}{cc}
\hline Melanoma Thickness (mm) & Excision Margin (cm) \\
\hline In situ & 0.5 \\
$\leq 1.0$ & 1.0 \\
$1.01-2.0$ & 1.0 to 2.0 \\
$2.01-4.0$ & 2.0 \\
$>4.0$ & At least 2.0 \\
\hline
\end{tabular}

nodes, thus decreasing regional recurrence. However, opponents say if SLN biopsy had been performed, there would then have been no difference in regional recurrence between the two groups. Any difference in the SLN positivity rate between the two groups would have been ascribed to uneven randomization, thereby causing any trend towards decreased survival. Had SLN biopsy been employed, conclusions of the trial would arguably have been the opposite (that there is no negative impact on either local recurrence or overall survival of 1-cm margins), and this approach may have become the standard of care for melanoma wide excisions.

To put an end to the conjecture, a sixth trial is needed: a randomized trial between 1 - and 2-cm margins for intermediate thickness melanoma. While such a trial has been proposed, it may not be practical because of the very large number of patients required to demonstrate equivalence. Whether or not that is appropriate is controversial, given the limited resources available for clinical research. However, without such a trial we are left wondering about the necessity for routine 2-cm margins. Is the resultant effect on aesthetics and the need for skin grafting over-treatment?

Based on the evidence described above, the recommended margins of excision are listed in Table 2. Technically, wide excision involves marking the proper radial margin circumferentially around the lesion or the site of the biopsy (Fig. 1). Margins should be measured in each direction from the periphery of the lesion or scar rather than from the center. This technique typically results in a circular or oval area of resection, which would be difficult to close primarily. Thus, an ellipse is created that incorporates this circle. The long axis is typically oriented to best facilitate primary closure, eg, longitudinally along an extremity. The long axis is often deter- mined by the orientation of the initial biopsy, which is why it is important to always plan for eventual wide radical excision when performing an excisional biopsy of an atypical skin lesion. Although a length to width ratio of approximately $3.5: 1$ or $4: 1$ is optimal for the ellipse, this ratio may result in an exceptionally long scar. A ratio of 3:1 is usually adequate to avoid "dog-earing," but this problem can also be corrected by the use of a V-plasty.

While the radial excision margin is important, the depth of excision must also be considered. Historically, some surgeons have believed that excising the underlying muscular fascia might promote the dissemination of tumor cells and increase the recurrence rate. ${ }^{11}$ No prospective trials have ever addressed this question. A retrospective trial ${ }^{12}$ published in 1982 found no significant difference in survival or local recurrence for patients in whom fascia was preserved versus patients in whom the fascia was removed. The current practice is excision of cutaneous and subcutaneous tissue down to, but not including, the underlying fascia. Some surgeons advocate removal of the underlying fascia en bloc when the previous surgical biopsy closely approaches or includes the underlying fascia.

Most wide excisions of primary melanoma can be closed primarily. Skin flaps are raised in the plane above the deep fascia to alleviate tension. Closure is performed in two layers. Interrupted deep dermal absorbable sutures should be followed by a running subcuticular stitch, or nylon sutures, depending on the skin tension and the anticipated motion effects. When the wound cannot be closed primarily, more complex coverage techniques must be employed such as skin grafting or use of local and distant flaps. If advancement flaps can be used, this is preferable to skin grafts, because patient acceptance of cosmesis has more to do with the soft tissue indentation than the length of the incision. ${ }^{13}$ If complex flaps may be necessary for wound coverage, preoperative planning with a plastic surgeon is warranted. Flaps can offer an excellent color match, durability, and overall cosmesis.

Skin grafting may be either split-thickness or full-thickness, depending on recipient location. The skin graft donor site should preferentially be outside the area of potential intransit metastases. Partial thickness skin grafts are obtained using a dermatome calibrated to harvest 0.12 to 0.18 inch,

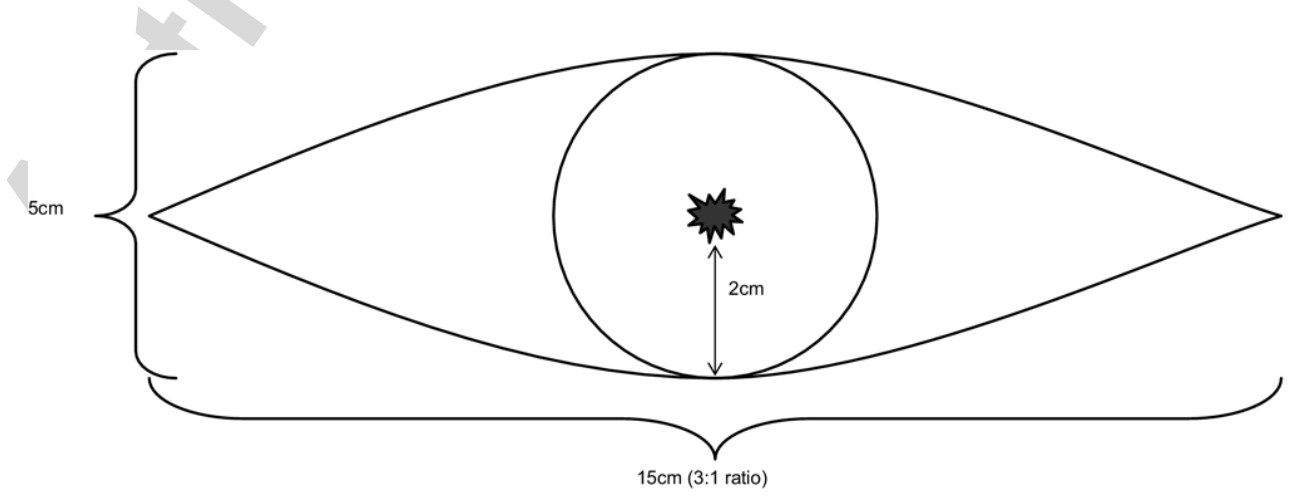

Figure 1 Wide excision of melanoma. Appropriate margins are measured around the melanoma or biopsy scar. An ellipse is then created that incorporates the area with a length to width ratio of at least 3 to 1 . Obtaining a 2-cm margin around a melanoma (or biopsy site) $1 \mathrm{~cm}$ in diameter results in a scar length of $15 \mathrm{~cm}$. 
which provides epidermis and partial dermis. The graft can be meshed, if necessary, to fill a large defect. If meshing is not performed, several small incisions should be made with a scalpel to prevent seroma formation underneath the graft. The graft is then secured to the wound by staples or sutures. It is critical that the entire surface of the graft adhere to the wound base for the first few days for the graft to survive. Stable adherence can be achieved with a gently compressive bolster and a splint for the extremity. The bolster is usually taken off to allow the graft to be examined 3 to 5 days after placement.

Full-thickness grafts encompass the entire epidermis and dermis, and they tend to contract less and give better cosmesis. For most patients with melanoma (even thin patients) adequate full-thickness grafts can be easily obtained from areas of redundant skin (supraclavicular, lower hypogastric, inguinal, axillary) to fill most resultant defects. The graft can often be obtained from the SLN biopsy region, thus precluding the need for a third incision. It is important to orient the donor site incision carefully so that it can be re-excised with a subsequent node dissection if the sentinel node is positive. Full-thickness grafts should be used in the head and neck region, but split-thickness skin grafts are preferred for the scalp. Skin grafts to the face should be carefully matched for color and texture.

Melanomas arising on the skin or nail bed of the toes are managed with a straightforward amputation at the metatarsophalangeal joint. Lesions that arise between two toes often require amputation of both adjoining toes. When melanomas arise on the fingers, surgeons attempt to preserve as much of the digit as possible without compromising margins. Typically, amputations are performed at the middle interphalangeal joint of the fingers or proximal to the distal joint of the thumb. One exception to this is the index finger, where amputation of the digit at the middle interphalangeal joint can interfere with the patient's grasp. A more cosmetically pleasing and more functional outcome can often be achieved with a Ray amputation, where the distal half of the metacarpal is removed as well.

\section{Lymph Node Dissection for Clinically Evident Regional Metastases}

All patients with melanoma should undergo a careful physical examination, with special attention being paid to the regional draining, lymph node basins. Between $5 \%$ and $10 \%$ of patients have clinical evidence of nodal metastases when first diagnosed with melanoma. ${ }^{14}$ Surgical excision of palpable regional lymph node disease can be potentially curative. In addition, resection of nodal disease can palliate pain and prevent skin breakdown.

When clinically suspicious nodes (generally $>1 \mathrm{~cm}$, hard or fixed to adjacent structures) are present in a patient with melanoma or a history of melanoma, fine-needle aspiration (FNA) biopsy is recommended, because it is highly accurate and does not interfere with the subsequent dissection. Open biopsy complications (seroma, infection, or hematoma) and the need to excise the resultant scar, may not only worsen the morbidity of the definitive operation but increase the risk of local recurrence..$^{15}$ Excisional biopsy should be reserved for cases where the FNA biopsy results are inconclusive. The biopsy incision should be oriented in a way that it can be easily incorporated into the resection margins. In addition, given the high likelihood of distant disease and the morbidity of the operation, a metastatic work-up should be initiated prior to surgery. At a minimum, patients should have a complete history and physical examination, chest radiograph, and serum lactate dehydrogenase with any abnormalities prompting a more thorough search. However, most surgeons advocate routine imaging in asymptomatic patients, consisting of either computerized tomography (CT) scans of the chest, abdomen and pelvis, 18 -flourodeoxyglucose positron emission tomography (FDG-PET), or a CT-PET study. In many cases these radiographs will upstage stage III patients to stage IV, which will alter the therapeutic approach. ${ }^{16-18}$

For patients with cervical metastases from epithelial malignancies, the gold standard has been the radical neck dissection to remove all ipsilateral cervical lymph nodes in levels $\mathrm{I}-\mathrm{V}$. The spinal accessory nerve, internal jugular vein, and sternocleidomastoid muscle are removed as well. However, several studies have demonstrated no significant differences in regional control of melanoma with the modified radical versus radical approaches. ${ }^{19-21}$ The modified radical neck dissection removes all lymph nodes routinely excised in a radical neck dissection but, at a minimum, preserves the spinal accessory nerve and attempts to preserve the internal jugular vein and sternocleidomastoid muscle. A parotidectomy and neck dissection are advocated for patients with parotid nodal involvement.

When palpable disease is evident in the axilla, axillary lymph node dissection (ALND) is used to remove levels I and II nodes completely and at least some level III nodes. The thoracodorsal neurovascular bundle and long thoracic nerve are spared. In the cases of minimal disease burden, it may be adequate to remove only levels I and II nodes. To access clinically involved level III nodes, it is often necessary to divide the pectoralis minor muscle. However, some surgeons believe clinically uninvolved level III nodes can be removed adequately by retraction of the pectoralis muscle without actual division. The intercostobrachial nerves may also be preserved, although most surgeons routinely resect these nerves when palpable disease is present, causing paresthesias of the upper inner arm. Brachial plexus injuries are rare but devastating complications, so great care is taken to avoid them.

A complete axillary dissection even in the most experienced hands carries significant morbidity. The most common long-term complication is lymphedema, which can be quite disabling and occurs to some degree in at least $10 \%$ of cases. ${ }^{22,23}$ Preserving the muscle fasciae from the pectoralis and latissimus dorsi muscles during the dissection, or avoiding skeletonization of the axillary vein (which some surgeons consider essential), may decrease lymphedema rates. $^{24}$ 
When disease is present in the groin, the extent of the node dissection is controversial. A superficial inguinal lymph node dissection (ILND) is performed by removing the fibrofatty node-bearing tissue inferior to the inguinal ligament and between the adductor muscle and the sartorius muscles. The tissue superficial to the external oblique fascia from the level of the anterior superior iliac spine laterally to the pubic tubercle medially also contains lymph nodes and is included in the dissection. When palpable nodal disease is present, some surgeons routinely excise the pelvic nodes. These nodes can be removed en bloc by incising the external oblique fascia, dividing the inguinal ligament, or making a separate midline laparotomy incision. This procedure is typically referred to as a deep dissection, which adds removal of the iliac nodes to the inguinofemoral nodes. Other surgeons take a more selective approach to a deep dissection. Some surgeons recommend that if the node between the inguinal and iliac chains (Cloquet's node) is removed and found to harbor metastatic disease, then a deep dissection should be performed en bloc with the superficial dissection. Unfortunately, the absence of disease in Cloquet's node does not always predict the absence of involvement of iliac nodes. ${ }^{25}$ Other selection criteria for deep dissection are enlarged pelvic nodes by CT scan and three or more metastatic lymph nodes found in the superficial dissection.

Whether the more extensive dissection confers a survival advantage remains unknown. In a retrospective review of 104 patients who underwent either combined superficial with deep dissection or superficial dissection only for palpable groin disease, the extent of surgery did not influence survival or local control rates. ${ }^{26}$ In another retrospective review of 227 patients having superficial versus superficial plus deep inguinal lymphadenectomy, the extent of surgery was not associated with a survival difference, and these authors recommended superficial plus deep dissection only in patients with clinical or radiologically positive deep nodal disease. ${ }^{27}$

The morbidity of groin dissection is more substantial than axillary dissection, with complication rates between $50 \%$ and $60 \% .7,28$ Wound complications are common with an ILND and include skin flap necrosis, wound infection, and dehiscence. Lymphedema rates are approximately 20\% to $30 \% .{ }^{29-31}$ Preservation of the saphenous vein or preservation of muscle fasciae from the adductor during dissection may also decrease the rate of lymphedema but is rarely feasible in the node-positive groin dissection. Most surgeons advocate use of 20 to $30 \mathrm{~mm} \mathrm{Hg}$ compression hose for several months postoperatively. These hose should be obtained preoperatively, when the extremity circumference is normal, rather than postoperatively when edema may have already partially developed.

\section{Regional Management of the Clinically Node-Negative Patient}

Most patients who present with primary melanoma have clinically negative (non-palpable) regional lymph nodes, but as many as $20 \%$ to $30 \%$ of these patients harbor occult regional nodal metastases. Before the description by Morton et $\mathrm{al}^{32}$ in 1992 of the technique of lymphatic mapping and SLN biopsy in melanoma cases, much controversy existed surrounding the management of the nodal basin. With the understanding that primary melanomas often spread to regional nodal basins before metastasizing widely, some surgeons advocated elective lymph node dissection (ELND), with the idea that early clearance of tumor deposits in the regional nodal basin could prevent subsequent dissemination and improve survival. However, given the significant morbidity of nodal clearance from regional nodal basins, there was much interest in knowing whether or not ELND provided true benefit.

While retrospective data suggested a survival benefit for ELND, ${ }^{33,34}$ four prospective trials failed to demonstrate this. ${ }^{35-38}$ However, some data suggested that there might be subsets of patients who did benefit. The Intergroup Melanoma Surgical Program randomized 740 stage I and II patients to ELND or observation. ${ }^{36}$ While there was no overall difference in survival between the two groups, in a subgroup analysis ELND conferred a survival benefit in patients with non-ulcerated melanomas and in patients with tumor thickness between 1 and $2 \mathrm{~mm}$. Further evidence comes from the WHO Melanoma Group Program 14 trial, which randomized patients with truncal melanoma to wide excision plus ELND or wide excision plus observation, with node dissection being done if patients subsequently recurred. ${ }^{37}$ Again, there was no overall survival benefit, but when survival of patients with microscopic disease on ELND were compared with those who had regional recurrences, the survival was significantly improved in the ELND group (48.2\% v 26.6\%, $P=.04$ ).

These data suggest, but do not prove, that node dissection will improve survival among those patients who harbor microscopic disease. There was no accurate way during these trials to identify such patients, but the introduction of lymphatic mapping and SLN biopsy has dramatically changed that point. The technique is a minimally invasive procedure that identifies patients with occult microscopic disease and is best performed at the time of wide local excision of the primary lesion. The hypothesis underpinning the technique is that melanoma involvement of a nodal basin develops in an orderly fashion with metastasis to the SLN as the first step in the process. ${ }^{39}$ Identification and removal of the SLN will accurately stage that nodal basin and, in turn, identify those patients who would benefit from full node dissection, while preventing node-negative patients from undergoing a substantially morbid procedure. ${ }^{40}$ The accuracy of the SLN in reflecting the pathologic status of the entire regional basin has been confirmed in multiple studies. ${ }^{39,41,42}$

There are two common methods for identifying the SLN: a blue dye ( $1 \%$ isosulfan blue, Lymphazurin, Tyco Healthcare, Norwalk, CT) and a radiolabeled colloid solution (99m technetium-labeled sulfur colloid). The radiolabeled colloid is injected 1 to 4 hours preoperatively, and the blue dye is injected intradermally at the site of the primary tumor a few minutes before the SLN biopsy incision is made. The surgeon then uses a hand-held gamma probe to mark the "hot spot" location of the SLN, thereby minimizing the skin incision 


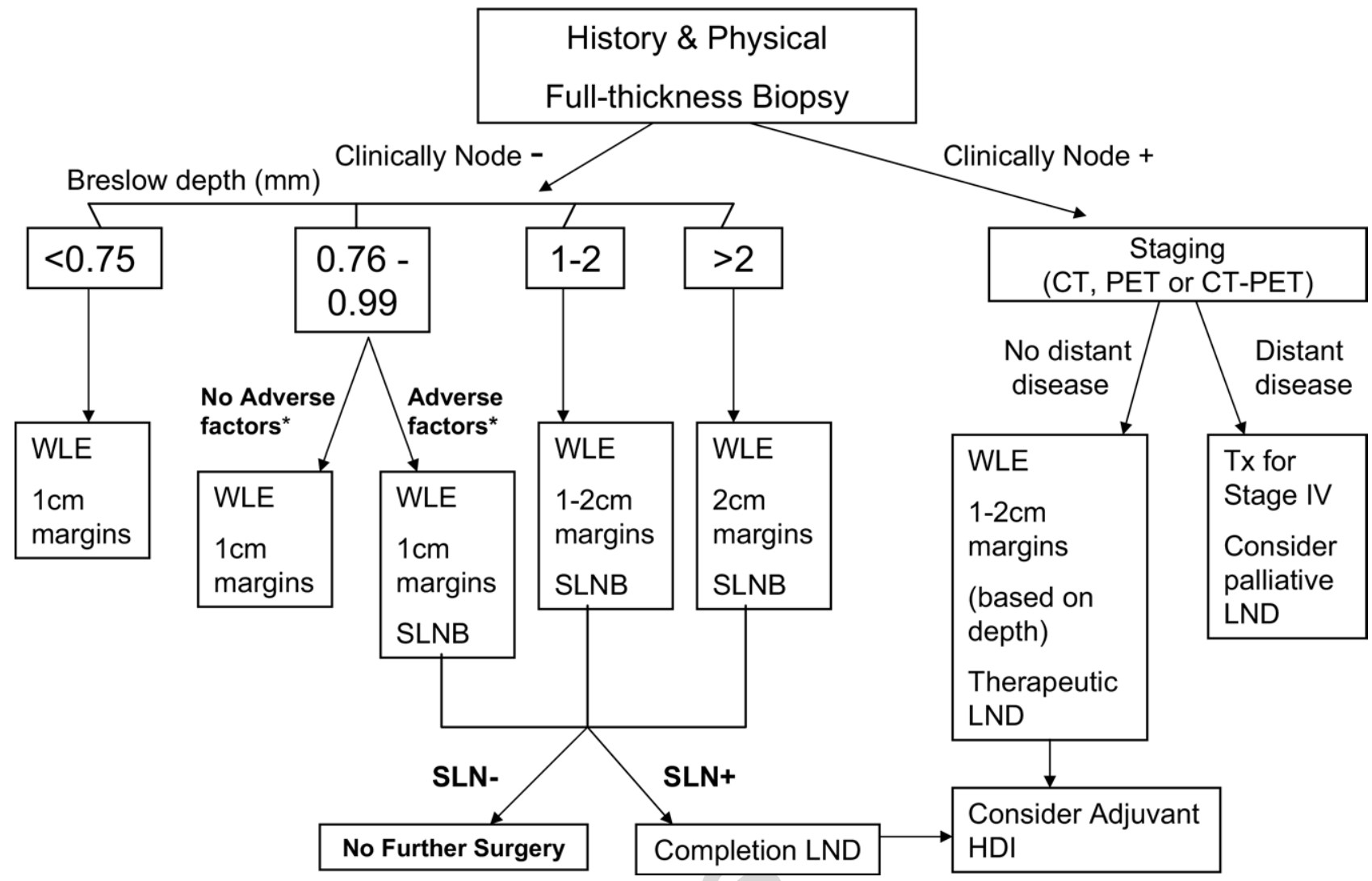

Figure 2 Surgical management of primary melanoma. *Adverse factors include angiolymphatic invasion, young patient age, high mitotic rate, ulceration, or Clark level IV or V. WLE, wide local excision; SLNB, sentinel lymph node biopsy; LND, lymph node dissection; HDI, high-dose interferon; Tx, treatment.

size. Once the incision is made, the surgeon identifies the SLN by either following blue-stained lymphatics or by finding the areas with the highest radioactive signal. The combined use of the blue dye and radiolabeled colloid correctly identifies the sentinel node in more than $95 \%$ of cases. Morbidity for lymphatic mapping and SLN biopsy is much lower than complete lymph node dissection.

In addition to preventing unnecessary lymphadenectomies, SLN biopsy also provides more accurate staging. The SLN undergoes serial step-sectioning for routine hematoxylin and eosin (H\&E) staining as opposed to simple bivalving the node, as is typical with full lymphatic dissections. If serial sectioning and $H \& E$ staining are negative for metastasis, then immunohistochemical staining for melanoma markers such as S-100, Melan-A, and HMB-45 is performed. With this increased sensitivity, SLN status is now the most important predictor of recurrent disease and survival for patients with melanoma.

Currently, SLN biopsy is considered the standard of care by most surgical oncologists for staging the regional lymph nodes of patients with primary cutaneous melanomas $>1.0$ $\mathrm{mm}$ thickness. Patients with thin melanomas $(<1.0 \mathrm{~mm})$ have a low incidence of regional metastases, so SLN biopsy is not routinely recommended. However, in some cases the presence of adverse prognostic features (ulceration, Clark level, high mitotic rate, young patient age, angiolymphatic invasion, or Clark's level IV or V tumors) may prompt SLN biopsy in patients with thin melanomas. ${ }^{43,44}$ The incorporation of SLN biopsy into the surgical therapy of melanoma is illustrated in (Fig. 2).

Does SLN biopsy with subsequent lymph node dissection improve outcome? This issue is being addressed by the Multicenter Selective Lymphadenectomy Trial I (MSLT-I), which randomized patients to wide excision alone or wide excision plus SLN biopsy, with complete lymph node dissection (CLND) for patients with a positive SLN. Interim results demonstrate that the 5-year survival for patients who had a CLND for a positive SLN (including false negative patients; those patients who had a regional recurrence despite a negative SLN) was significantly better than for those patients undergoing CLND for a recurrence after wide excision alone (66.2\% v 54.2\%; HR 0.62; 95\% CI, .40 to .95; $P<.02) .{ }^{45}$ This result provides further evidence that CLND may improve survival for the subset of patients with occult metastases. Final results from the MSLT-I trial are still pending.

A still unresolved question is how much the subsequent CLND benefits the patient over and above the SLN biopsy itself. In many patients the SLN will be the only nodal site in which disease is identified pathologically and is thus therapeutic by itself. Approximately 70\% to $90 \%$ of patients have no detectable disease in the non-sentinel nodes removed at the time of dissection, although "non-sentinel" nodes are not subjected to the same intense pathologic scrutiny as are the sentinel nodes. While there is much interest in identifying 
Table 3 Melanoma TNM Classification

\begin{tabular}{cll}
\hline T Classification & \multicolumn{1}{c}{ Thickness (mm) } & \multicolumn{1}{c}{ Ulceration Status } \\
\hline T1 & $\leq 1.0$ & $\begin{array}{l}\text { a: Without ulceration and level II/III } \\
\text { b: With ulceration or level IV/V }\end{array}$ \\
T2 & $1.01-2.0$ & $\begin{array}{l}\text { a: Without ulceration } \\
\text { b: With ulceration }\end{array}$ \\
T3 & $2.01-4.0$ & a: Without ulceration \\
b: With ulceration & a: Without ulceration \\
T4 & $>4.0$ & b: With ulceration
\end{tabular}

Abbreviations: LDH, lactate dehydrogenase; TNM, tumor, node, metastasis.

*Micrometastases are diagnosed after sentinel or elective lymphadenectomy.

tMacrometastases are defined as clinically detectable nodal metastases confirmed by therapeutic lymphadenectomy or nodal metastasis that exhibits gross extracapsular extension.

those patients that may not require CLND, predicting which patients will have residual disease has proven unreliable. ${ }^{46-48}$ This question is being prospectively addressed in the Multicenter Selective Lymphadenectomy Trial II (MSLT-II), which randomizes patients with a positive SLN to CLND or observation with serial ultrasounds of the regional node basin.

\section{Satellite Lesions and In-Transit Metastases}

In- transit metastases and satellitosis develop in 5\% to $8 \%$ of patients with melanoma thickness greater than $1.5 \mathrm{~mm} .{ }^{49}$ Historically, satellite lesions were defined as skin involvement within $2 \mathrm{~cm}$ of the primary lesion, whereas in-transit metastases were lesions between the primary tumor and a regional lymph node basin but greater than $2 \mathrm{~cm}$ from the primary tumor. These lesions were considered and treated separately. However, Singletary et a ${ }^{50}$ demonstrated in a retrospective review of 135 patients with regional cutaneous metastases that classifying the lesions as satellites or in-transit metastases on the basis of distance from primary tumor had no prognostic significance. Both portend a poor prognosis. The current American Joint Committee on Cancer (AJCC) staging system classifies both as Stage III disease, and we will consider these lesions together (Table 3). Surgical therapies for in-transit disease primarily involve three strategies: local excision or ablative therapies, isolated limb perfusion (ILP), or isolated limb infusion (ILI).

When the number of lesions is small and the affected area confined, optimal management is complete surgical excision. ${ }^{51}$ A specific margin of excision is not required, but histologically complete removal of the metastases should be the goal. Because distant metastatic disease is often present in patients with in-transit disease, a metastatic work-up before resection is recommended. When the number or location of the in-transit lesions preclude surgical resection, intralesional therapies may be employed, but the response rates are low and their durations generally short. ${ }^{49,52}$ Given the availability of extremity perfusion strategies, intralesional therapies are more frequently used for lesions on the trunk or head and neck. Other local treatment modalities such as radiotherapy, cryotherapy, electrodessication, and laser ablation also have been used and have been effective in selected situations. ${ }^{53}$

When the number of lesions is beyond surgical resection in the extremity, patients may be candidates for ILP. ILP consists of regional administration of high-dose chemotherapy (doses up to 15 to 25 times higher than systemic therapy) within an extremity using an extracorporeal membrane oxygenator similar to cardiac surgery. ${ }^{51}$ Isolation of the vessels is obtained surgically, a tourniquet is applied to prevent leakage of chemotherapy systemically. Using the bypass machine, 
Table 4 Survival After Complete Resection of Skin and Soft Tissue Metastases

\begin{tabular}{clc}
\hline First Author and Year & \multicolumn{1}{c}{ Site (N) } & $\begin{array}{c}\text { 5-Year } \\
\text { Survival }\end{array}$ \\
\hline Markowitz, 199163 & Soft tissue (60) & $49 \%$ \\
& Lymph nodes (72) & $38 \%$ \\
Gadd, 199264 & Skin, soft tissue, & $14 \%$ \\
& lymph nodes (190) & \\
Karakousis, 1995 & Lymph nodes (23) & $22 \%$ \\
& Subcutaneous (27) & $33 \%$ \\
Meyer, 200066 & Lymph nodes (45) & $20 \%$ \\
& Subcutaneous (30) & $18 \%$ \\
\hline
\end{tabular}

the limb is heated to $38-40^{\circ} \mathrm{C}$ with high concentration chemotherapy perfusate. After the treatment, which usually lasts 60 to 90 minutes, the drug is flushed from the circulation, and systemic circulation is restored. The most common chemotherapeutic agent employed has been melphalan. Using this regimen, overall response rates between $80 \%$ and $90 \%$ and complete response rates between $55 \%$ and $65 \%$ are obtained. ${ }^{51}$ The duration of response is typically 9 to 12 months, but a subgroup of complete responders (which is $20 \%$ to $25 \%$ of the total patient population) can have sustained complete responses. Toxicities range from mild erythema and edema to extensive epidermolysis, functional impairment, and sometimes even a need for amputation.

ILI is a less invasive and less toxic strategy for management of in-transit metastases of the extremity; this technique was developed in Australia approximately 12 years ago. ${ }^{53,54}$ Access is gained to the circulation of the affected limb by standard percutaneous radiologic techniques. Arterial and venous catheters are then connected to a simple extracorporeal circuit incorporating a heating coil. A tourniquet is inflated around the proximal limb. The chemotherapeutic agent is then infused into the isolated limb, although at lower doses than those used with ILP because there will be some systemic leakage. In one series using melphalan and dactinomycin, the overall response in limbs treated by ILI was $85 \%$, with a complete response of $41 \%$ and a partial response of $44 \%$. Median duration of response was 16 months, results that compare favorably to the more invasive and complex technique of ILP. ${ }^{54}$ Currently, ILI is being further evaluated in phase II trials.

\section{Surgical Management of Metastatic Melanoma}

Up to one third of patients with melanoma develop distant metastatic disease with limited options for therapy and a poor prognosis, with a 5 -year survival rate of less than $10 \%$. While surgery is rarely considered for most patients with solid tumor metastases, melanoma remains an exception, with documented long-term survivals among some patients who undergo resection of metastatic lesions. While most patients will not be candidates for resection, selected patients should be considered for surgery.
Careful selection for surgery of patients with stage IV melanoma is imperative. The decision should be made on the locations of the metastases, the number of lesions, morbidity of the operation(s), the disease-free interval, and the overall health and prognosis of the patient. Before surgery, the physician should carefully search for additional metastatic disease by performing the following: a thorough history and physical examination, serum lactate dehydrogenase, CT scans, magnetic resonance imaging (MRI) of the brain, and/or a FDG-PET scan.

\section{Soft Tissue Metastases}

The most common site of distant melanoma metastases are remote areas of skin and soft tissue, including lymph nodes outside of the draining basins. These patients have better outcomes compared to those with visceral metastases, with a 5-year survival of $19 \%$. There should be no hesitancy to resect isolated dermal or subcutaneous metastases if technically feasible, because these patients may have a reasonable long-term prognosis; 5-year survivals of $15 \%$ to $50 \%$ have been described (Table 4). Because resection may also be palliative, erring on the side of an aggressive surgical approach is often reasonable. Patients with more extensive soft tissue disease should be evaluated on an individual basis.

\section{Lung Metastases}

Fifteen to 30\% of melanoma metastases will occur in the lungs. Several reported series have demonstrated the feasibility of, and the improved survival from, pulmonary metastasectomy in this setting (Table 5). In a series of 945 patients with pulmonary metastatic melanoma from the Duke University Medical Center, 99 underwent partial or total resection. .55 Survival was significantly better after complete pulmonary resection than with no resection. For isolated pulmonary metastases, the 5-year survival rate was $4 \%$ for patients who did not undergo resection and increased to $20 \%$ for those who underwent complete metastasectomy. At the M.D. Anderson Cancer Center 56 patients underwent pulmonary metastasectomy ${ }^{56}$ Actuarial survival was $25 \%$ at 5 years with a median survival of 18 months.

\section{Liver Metastases}

Cutaneous melanoma metastasizes to the liver in 15\% to $0 \%$ of patients with stage IV disease. The role of surgical resection

Table 5 Survival After Complete Resection of Pulmonary Metastases

\begin{tabular}{|c|c|c|}
\hline First Author and Year & $\mathbf{N}$ & $\begin{array}{l}\text { 5-Year } \\
\text { Survival }\end{array}$ \\
\hline Wong, $1988^{67}$ & 38 & $31 \%$ \\
\hline Gorenstein, $1991^{56}$ & 56 & $25 \%$ \\
\hline Harpole, $1992^{55}$ & 98 & $20 \%$ \\
\hline Karakousis, $1995^{65}$ & 39 & $14 \%$ \\
\hline Tafra, $1995^{68}$ & 106 & $27 \%$ \\
\hline La Hei, $1996^{69}$ & 83 & $22 \%$ \\
\hline Leo, $2000^{70}$ & 282 & $22 \%$ \\
\hline
\end{tabular}


for such patients is controversial. Some have argued that the prognosis of metastatic disease to the liver is so dismal resection of metastases is not worthwhile. However, a recent multi-institutional retrospective study ${ }^{57}$ of hepatic resection for metastatic melanoma with curative intent evaluated 40 patients with cutaneous $(n=24)$ or ocular melanoma $(n=$ 16). Tumor recurred in the liver or systemically in $75 \%$ of these patients. There were no long-term survivors in patients with cutaneous melanoma, but the 5-year survival of patients with metastatic ocular melanoma was $20.5 \% .^{57}$ These data are limited by lack of appropriate controls but suggest that properly selected patients may benefit from resection, given the dismal median survival (only 2 to 7 months) of patients with hepatic melanoma metastases. Historically, only about $2 \%$ of patients with metastatic melanoma to the liver have been candidates for surgical resection. However, liver surgery results have improved significantly over the past decade, causing expansion of the criteria for resection. As regionally directed therapy options to the liver expand, surgery for management of patients with melanoma metastatic to the liver may play a greater role.

\section{Gastrointestinal Tract Metastases}

In one large series, resection of melanoma from the gastrointestinal (GI) tract represented 16\% of all metastasectomies. GI tract metastases are often associated with disseminated disease and, when documented, the median survival is usually only 5 to 11 months. These metastases often present with symptoms including abdominal pain, bleeding, or obstruction, and surgical intervention is clearly indicated for palliation of these problems. However, in some of these cases a survival advantage may also be realized. In one retrospective review $^{58}$ of 124 patients who presented with symptomatic GI tract metastases (metastatic melanoma to the stomach, small intestine, colon, or rectum), 69 underwent surgical exploration and, of these, 46 underwent curative resection and 23 had a palliative procedure. Palliation of symptoms was excellent. The median survival in patients undergoing curative resection was 48.9 months, compared to only 5.4 months and 5.7 months in those undergoing palliative procedures and nonsurgical interventions, respectively. In other series, long-term survivors up to 20 years have been reported when undergoing surgical excision of a solitary GI metastasis. ${ }^{59-62}$

\section{Brain and Spinal Cord Metastases}

Up to $60 \%$ of patients with metastatic melanoma develop clinically evident brain metastases at some time in their course. Brain metastases account for $20 \%$ to $54 \%$ of the deaths from melanoma and, when untreated, median survival is only 1 to 2 months. ${ }^{1}$ Surgical excision is favored over whole brain irradiation for larger $(>3.5 \mathrm{~cm})$ symptomatic metastases and may be sometimes indicated when several foci of metastatic disease are present. Stereotactic radiosurgery has gained popularity for treating smaller $(<3 \mathrm{~cm})$ lesions. Surgical excision of brain metastases may improve survival up to an average of 6 months, but the main role of surgery in this grim situation remains symptom control, not cure. Long-term success is very uncommon.

In summary, for stage IV melanoma, selected patients with limited sites and numbers of metastases should be considered for complete surgical resection regardless of the location of the disease. In general, patients with non-visceral metastatic disease (skin and subcutaneous metastases and distant nodal disease) amenable to resection fare better than patients with visceral disease (lung, liver, GI tract). Patients with single versus multiple lesions, and patients with longer disease-free intervals between the primary tumor and the development of stage IV disease also fare better.

\section{Conclusions}

Surgery plays an important role in the management of melanoma at all stages. Surgery for early-stage disease is not only therapeutic, but with the advent of sentinel lymph node biopsy, is instrumental in the staging of disease. Surgery is also indicated in selected patients with advanced regional or distant disease for palliative purposes and occasionally curative effect. As new chemotherapeutic agents, biologic agents, and immunotherapies emerge and demonstrate their potential, the objectives and indications for surgery will certainly change, but until that time, surgical therapy remains the cornerstone of therapy for malignant melanoma.

\section{References}

1. Jemal A, Seigel R, Ward E, et al: Cancer Statistics, 2006. CA Cancer J Clin 56:8-106-130, 2006

2. Aloia TA, Gershenwald JE: Management of early-stage cutaneous melanoma. Curr Probl Surg 42:460-534, 2005

3. Chang AE, Johnson TM, Rees R: Cutaneous neoplasms, in Greenfield LJ, Mulholland MW, Oldham KT, et al (eds): Surgery: Scientific Principles and Practice. Philadelphia, Lippincott-Raven, 1997, pp 22312246

4. Essner R: Surgical treatment of malignant melanoma. Surg Clin North Am 83:109-156, 2003

5. Veronesi U, Cascinelli N: Narrow excision (1-cm margin). A safe procedure for thin cutaneous melanoma. Arch Surg 126:438-441, 1991

6. Karakousis CP, Balch CM, Urist MM, et al: Local recurrence in malignant melanoma: Long-term results of the multi-institutional randomized surgical trial. Ann Surg Oncol 3:446-452, 1996

7. Balch CM, Soong S-J, Ross MI, et al: Long-term results of a multiinstitutional randomized trial comparing prognostic factors and surgical results for intermediate thickness melanomas (1.0 to $4.0 \mathrm{~mm}$ ). Ann Surg Oncol 7:87-97, 2000

8. Cohn-Cedermark G, Rutqvist LE, Andersson R, et al: Long term results of a randomized study by the Swedish Melanoma Group on $2 \mathrm{~cm}$ vs $5 \mathrm{~cm}$ resection margins for patients with cutaneous melanoma with a tumor thickness of 0.8-2.0mm. Cancer 89:1495-1501, 2000

9. Khayat D, Rixe O, Martin G, et al: Surgical margins in cutaneous melanoma ( $2 \mathrm{~cm}$ versus $5 \mathrm{~cm}$ for lesions measuring less than $2.1 \mathrm{~mm}$ thick). Cancer 97:1941-1946, 2003

10. Thomas JM, Newton-Bishop J, A'Hern R, et al: Excision margins in high-risk malignant melanoma. N Engl J Med 350:757-766, 2004

11. Olsen G: Some views on the treatment of melanomas of the skin. Arch Chirurg Neerl 22:79-90, 1970

12. Kennedy DE, Brown BW, McBride CM: Excision of underlying fascia with a primary malignant melanoma: Effect on recurrence and survival rates. Surgery 92:615-618, 1982

13. Cassileth BR, Lusk EJ, Tenaglia AN: Patients' perceptions of the cos- 
metic impact of melanoma resection. Plast Reconstr Surg 71:73-75, 1983

14. de Braud F, Khayat D, Kroon BB, et al: Malignant melanoma. Oncol Hematol 47:35-63, 2003

15. Nathansohn N, Schachter J, Gutman H: Patterns of recurrence in patients with melanoma after radical lymph node dissection. Arch Surg 140:1172-1177, 2005

16. Tyler DS, Onaitis M, Kherani A, et al: Positron emission tomography scanning in malignant melanoma. Cancer 89:1019-1025, 2000

17. Acland KM, O'Doherty MJ, Russell-Jones R, et al: The value of positron emission tomography scanning in the detection of subclinical metastatic melanoma. J Am Acad Dermatol 42:606-611, 2000

18. Eigtved A, Andersson AP, Dahlstrom K, et al: Use of flourine-18-flourodeoxyglucose positron emission tomography in the detection of silent metastases from malignant melanoma. J Nucl Med 27:70-75, 2000

19. Byers RM: Treatment of the neck in melanoma. Otolaryngol Clin North Am 31:833-839, 1986

20. O'Brian CJ, Petersen-Schaefer K, Ruark D, et al: Radical, modified and selective neck dissection for cutaneous malignant melanoma. Head Neck 17:232-241, 1995

21. Van de Vrie W, Eggermont AMM, Van Putten WL, et al: Therapeutic lymphadenectomy in melanomas of the head and neck. Head Neck 15:377-381, 1993

22. Karakousis CP, Hena MA, Emrich LJ, et al: Axillary node dissection in malignant melanoma: Results and complications. Surgery 108:10-17, 1990

23. Wrightson WR, Wong SL, Edwards MJ, et al: Complications associated with sentinel lymph node biopsy for melanoma. Ann Surg Oncol 10: 676-680, 2003

24. Lawton $G$, Rasque H, Ariyan S: Preservation of muscle fascia to decrease lymphedema after complete axillary and ilioinguinofemoral lymphadenectomy for melanoma. J Am College Surg 191:16-23, 2002

25. Shen P, Conforti AM, Essner R, et al: Is the node of Cloquet the sentinel node for the iliac/obturator node group? Cancer 6:93-97, 2000

26. Kretschmer L, Neumann C, Preusser KP, et al: Superficial inguinal and radical ilioinguinal lymph node dissection in patients with palpable melanoma metastases to the groin: An analysis of survival and local recurrence. Acta Oncol 40:72-78, 2001

27. Mann GB, Coit DG: Does the extent of operation influence the prognosis in patients with melanoma metastatic to inguinal nodes? Ann Surg Oncol 6:263-271, 1999

28. Coit DG, Peters M, Brennan MF: A prospective randomized trial of perioperative cefazolin treatment in axillary and groin dissection. Arch Surg 126:1366-1372, 1991

29. Karakousis CP, Driscoll DL, Rose B, et al: Groin dissection in malignant melanoma. Ann Surg Oncol 1:271-277, 1994

30. Karakousis CP, Emrich LJ, Driscoll DL, et al: Survival after groin dissection for malignant melanoma. Surgery 109:119-126, 1991

31. Kissin MW, Simpson DA, Easton D, et al: Prognostic factors related to survival and groin recurrence following therapeutic lymph node dissection for lower limb malignant melanoma. Br J Surg 74:1023-1026, 1987

32. Morton DL, Wen DR, Wong JH, et al: Technical details of intraoperative lymphatic mapping for early stage melanoma. Arch Surg 127:392-399, 1992

33. Balch CM, Cascinelli N, Milton GW: Elective node dissection: Pros and cons, in Balch CM, Milton GW (edds): Cutaneous Melanoma: Clinical Management and Treatment Results Worldwide. Philadelphia, Lippincott, 1985, pp 131-157

34. Balch CM: The role of elective lymph node dissection in melanoma: rationale, results and controversies. J Clin Oncol 6:163-172, 1988

35. Veronesi U, Adamus J, Bandiera DC, et al: Delayed regional lymph node dissection in stage I melanoma of the skin of the lower extremities. Cancer 49:2420-2430, 1982

36. Balch CM, Soong S-J, Bartolucci AA, et al: Efficacy of an elective regional lymph node dissection of 1 to $4 \mathrm{~mm}$ thick melanomas for patients 60 years of age and younger. Ann Surg 224:255-266, 1996

37. Cascinelli N, Morabito A, Santinami M, et al: Immediate or delayed dissection of regional nodes in patients with melanoma of the trunk: A randomized trial. Lancet 351:793-796, 1998

38. Sim FH, Taylor WF, Pritchard DJ, et al: Lymphadenectomy in the management of stage I malignant melanoma: A prospective randomized study. Mayo Clin Proc 61:697-705, 1986

39. Morton DL, Thompson JF, Essner R, et al: Validation of the accuracy of intraoperative lymphatic mapping and sentinel lymphadenectomy for early-stage melanoma: A multicenter trial. Multicenter Selective Lymphadenectomy Trial Group. Ann Surg 230:453-463, 1999

40. Gershenwald JE, Colome MI, Lee JE, et al: Patters of recurrence following a negative sentinel lymph node biopsy in 243 patients with stage I or II melanoma. J Clin Oncol 16:2253-2260, 1998

41. Vuylsteke RJCLM, van Leeuwen PAM, Muller MGS, et al: Clinical outcome of stage I/II melanoma patients after selective sentinel lymph node dissection: Long-term follow-up results. J Clin Oncol 21:10571065, 2003

42. Thompson JF, McCarthy WH, Bosch CM, et al: Sentinel lymph node status as an indicator of the presence of metastatic melanoma in regional lymph nodes. Melanoma Res 5:255-260, 1995

43. Sondak VK, Taylor JMG, Sabel MS, et al: Mitotic rate and younger age are predictors of sentinel lymph node positivity: Lessons learned from the generation of a probabilistic model. Ann Surg Oncol 11:247-258, 2004

44. Bleicher RJ, Essner R, Foshag LJ, et al: Role of sentinel lymphadenectomy in thin invasive cutaneous melanomas. J Clin Oncol 21:13261331,2003

45. Morton DL, Thompson JF, Cochran AJ, et al: Sentinal-node biopsy or nodal observation in melanoma. N Engl J Med 355:1307-1371, 2006

46. Sabel MS, Griffith KA, Sondak VK, et al: Predictors of nonsentinel lymph node positivity in patients with a positive sentinel node for melanoma. J Am Coll Surg 201:37-47, 2005

47. Wagner JD, Gordon MS, Chuang TY, et al: Predicting sentinel and residual lymph node basin disease after sentinel lymph node biopsy for melanoma. Cancer 89:453-462, 2000

48. McMasters KM, Wong SL, Edwards MJ, et al: Frequency of nonsentinel lymph node metastasis in melanoma. Ann Surg Oncol 9:137-141, 2002

49. Eggermont AMM, van Geel AN, de Wilt JH, et al: The role of isolated limb perfusion for melanoma confined to the extremities. Surg Clin North Am 83:371-384, 2003

50. Singletary SE, Tucker SL, Boddie AW: Multivariate analysis of prognostic factors in regional cutaneous metastases of extremity melanoma. Cancer 61:1437-1440, 1988

51. Fraker DL: Management of in-transit melanoma of the extremity with isolated limb perfusion. Curr Treat Options Oncol 5:173-184, 2004

52. Si Z, Hersey P, Coates AS: Clinical responses and lymphoid infiltrates in metastatic melanoma following treatment with intralesional GM-CSF. Melanoma Res 6:247-255, 1996

53. Thompson JF, Kam PC: Isolated limb infusion for melanoma: A simple but effective alternative to isolated limb perfusion. J Surg Oncol 88:1-3, 2004

54. Thompson JF, Kam PC, Waugh RC, et al: Isolated limb infusion with cytotoxic agents: a simple alternative to isolated limb perfusion. Semin Surg Oncol 14:238-247, 1998

55. Harpole DH, Johnson CM, Wolfe WG, et al: Analysis of 945 cases of pulmonary metastatic melanoma. J Thorac Cardiovasc Surg 103:743748, 1992

56. Gorenstein LA, Putnam JB, ANatarajan G, et al: Improved survival after resection of pulmonary metastses from malignant melanoma. Ann Thorac Surg 52:204-210, 1991

57. Pawlik TM, Zorzi D, Abdalla EK, et al: Hepatic resection for metastatic melanoma: Distinct patterns of recurrence and prognosis for ocular versus cutaneous disease. Ann Surg Oncol 13:712-720, 2006

58. Ollila DW, Essner R, Wanek LA, et al: Surgical Resection for melanoma metastatic to the gastrointestinal tract. Arch Surg 131:975-979, 1996

59. Klasse JM, Kroon BB: Surgery for melanoma metastatic to the gastrointestinal tract. Br J Surg 77:60-61, 1990

60. Caputy GG, Donahue JH, Goellner JR, et al: Metastatic melanoma of the 
gastrointestinal tract. Results of surgical management. Arch Surg 126:336-344, 1991

61. Ricaniadis N, Konstadoulakis MM, Walsh D, et al: Gastrointestinal metastases from malignant melanoma. Surg Oncol 4:105-110, 1995

62. Agrawal S, Yao TJ, Coit DG: Surgery for melanoma metastatic to the gastrointestinal tract. Arch Surg 131:975-979, 1999

63. Markowitz JS, Cosimi LA, Carey RW, et al: Prognosis after initial recurrence of cutaneous melanoma. Arch Surg 126:703-707, 1991

64. Gadd MA, Coit DG: Recurrence patterns and outcome in 1019 patietns undergoing axillary or inguinal lymphadenectomy for melanoma. Arch Surg 127:1412-1416, 1992

65. Karakousis CP, Velez A, Driscoll BA, et al: Metastatectomy in malignant melanoma. Surgery 115:295-302, 1995
66. Meyer T, Merkel S, Goehl J, et al: Surgical therapy for distant metastases of malignant melanoma. Cancer 89:1983-1991, 2000

67. Wong JH, Euhus DM, Morton DL: Surgical resection for metastatic melanoma to the lung. Arch Surg 123:1091-1095, 1988

68. Tafra L, Dale PS, Wanek LA, et al: Resection and adjuvant immunotherapy for melanoma metastatic to the lung and thorax. J Thorac Cardiovasc Surg 110:119-128, 1995

69. La Hei ER, Thompson JF, McCaughan BC, et al: Surgical resection of pulmonary metastatic melanoma: A review of 83 thoracotomies. Asia Pacific Heart J 5:111-114, 1996

70. Leo F, Cagini L, Rocmans P, et al: Lung metastases from melanoma: When is surgical treatment warranted? Br J Cancer 83:569-557, 2000 